\title{
ARTICLE
}

\section{Altered frontostriatal white matter microstructure is associated with familial alcoholism and future binge drinking in adolescence}

\author{
Scott A. Jones ${ }^{1}$ and Bonnie J. Nagel ${ }^{1,2}$
}

\begin{abstract}
Adolescence is a time of significant neurobiological development, including changes in white matter microstructure. Familial alcoholism and adolescent binge-drinking have both been associated with altered white matter microstructure; however, the temporal nature of these effects, and their interaction, is unclear. Using diffusion-weighted imaging and voxel-wise multilevel modeling, the effects of familial alcoholism and future binge-drinking on white matter microstructural development were assessed in 45 adolescents, who went on to binge-drink (but were alcohol-naive at baseline), and 68 adolescents, who remained largely alcohol-naive, all with varying degrees of familial alcoholism. Both future binge-drinking and familial alcoholism were associated with altered frontostriatal white matter microstructure early in adolescence, prior to alcohol use. While several binge-drinkingrelated effects persisted throughout adolescence (in the posterior limb of the internal capsule, superior corona radiata, and cerebellar peduncles), the association between familial alcoholism and altered white matter microstructure dissipated across adolescence in all regions. There were no white matter regions identified where future binge-drinking or familial alcoholism were significantly associated with emergent or exacerbated alterations in white matter microstructure. Altogether, these findings suggest that alterations in frontostiatal white matter microstructure, some of which are associated with familial alcoholism, may be used to predict which adolescents are more likely to go on and engage in alcohol use. Meanwhile, a reduction in family history-related associations with altered white matter microstructure by late-adolescence is encouraging for future prevention work targeted at atrisk youth.
\end{abstract}

Neuropsychopharmacology (2019) 44:1076-1083; https://doi.org/10.1038/s41386-019-0315-x

\section{INTRODUCTION}

Adolescent neurodevelopment is highlighted by extensive volumetric change in both gray and white matter [1,2]. Underlying increases in white matter volume are changes in the microstructural properties of white matter fibers. With diffusionweighted imaging (DWI), widespread increases in fractional anisotropy (FA), thought to reflect greater fiber density, axonal diameter, and myelination, and decreases in mean diffusivity (MD), thought to reflect greater white matter density [3, 4], have been repeatedly demonstrated across adolescence $[2,5]$. These changes in white matter microstructure with age, particularly in frontostriatal regions, have been associated with several developing executive processes, including lower impulsivity [6], greater inhibitory control [7], and greater working memory capacity [8], highlighting the relevance of white matter microstructure as a neurobiological marker for cognitive maturation in adolescents.

Both personal and familial alcohol misuse have been associated with alterations in white matter microstructure during adolescence. Binge-drinking, the most common form of alcohol misuse during adolescence, has been associated with widespread reductions in FA [9-12]; however, in regions such as the superior longitudinal fasciculus and internal capsule, greater FA has been reported in binge-drinking adolescents compared to controls [9].
Reports of altered MD in binge-drinking adolescents are less common, and have produced mixed findings, suggesting both greater and lower MD in binge-drinking adolescents compared to controls $[9,11,12]$. Meanwhile, adolescents free of personal alcohol use, but with a family history $(\mathrm{FH})$ of alcoholism, also demonstrate both greater and lower FA, and lower MD [13, 14], including in frontostriatal regions important for reward-based decision making, such as the decision to drink. Given that $\mathrm{FH}$ is a significant predictor of personal alcohol misuse [15], this begs the question of whether alterations in white matter microstructure evident in binge-drinking adolescents are present prior to alcohol use and represent a developmentally transient or sustained predisposition associated with $\mathrm{FH}$ or are consequences of alcohol use itself. Further, it is also possible that alterations in FA related to $\mathrm{FH}$ precede binge drinking and are further exacerbated by alcohol use.

Longitudinal studies in binge-drinking adolescents suggest that those who go on to drink demonstrate significant FA decreases and $M D$ increases with time, including in frontostriatal white matter regions, when compared to adolescents who remain alcohol-naive, despite having comparable levels of FA and MD prior to alcohol use $[10,11]$. Conversely, associations between $\mathrm{FH}$ and altered FA appear to be greater in adolescents than young

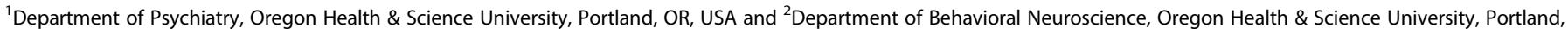
OR, USA

Correspondence: Bonnie J. Nagel (nagelb@ohsu.edu)

Received: 20 September 2018 Revised: 20 December 2018 Accepted: 7 January 2019

Published online: 12 January 2019 
adults [16], and a recent longitudinal study suggests that the association between white matter microstructure and $\mathrm{FH}$ of psychopathology (including familial alcoholism) is largely transient [17]. While a previous cross-sectional study (utilizing an a priori region of interest) found that $\mathrm{FH}$ may interact with personal alcohol use to predict more severe reductions in FA in the superior and inferior longitudinal fasciculus [18], no study has investigated the role of $\mathrm{FH}$ in the development of white matter microstructure in drinking and non-drinking adolescents.

Given that both personal and familial alcohol use have independently been associated with alterations in frontostriatal white matter microstructure in regions implicated in executive control processes, including reward-based decision making $[6,14]$, more nuanced investigations are necessary to elucidate the interaction between personal and familial alcohol misuse on maturation of this neurocircuitry. Thus, the current study aimed to investigate the association of future binge-drinking status and $\mathrm{FH}$ of alcoholism on white matter microstructural development. Based on previous findings, we hypothesized that future binge-drinking adolescents would show an altered course of white matter maturation, characterized by age-related reductions in FA and increases in MD in frontostriatal white matter regions compared to alcohol-naive controls. Further, it was hypothesized that $\mathrm{FH}$ of alcoholism would be associated with greater reductions in FA and greater increases in MD with age in future binge-drinking adolescents, but would only be associated with altered white matter microstructure early in adolescence in controls.

\section{MATERIALS AND METHODS}

Recruitment and exclusionary criteria

Participants were recruited from the community as part of an ongoing longitudinal study on adolescent neurodevelopment $[19,20]$. This study included neuroimaging data from 113 adolescents (ages 11-16 at baseline), a subset of which were used in an analysis investigating the effects of familial psychopathology on white matter microstructural development [17]. During recruitment, adolescents and their parents provided written consent and assent, respectively, and completed comprehensive screening interviews. Exclusionary criteria included: probable diagnosis of a DSM-IV psychiatric disorder, including mental retardation or learning disability [21], inability to obtain family history information, serious medical problems (including head trauma), psychotic illness in a biological parent, known prenatal drug/alcohol exposure, left-handedness [22], and MRI contraindications. Furthermore, as the goals of the study were to examine premorbid white matter microstructural alterations associated with $\mathrm{FH}$ of alcoholism prior to alcohol use, as well as the effects of personal alcohol use on white matter maturation, adolescents were excluded for baseline drug and alcohol use that exceeded $>10$ lifetime alcohol drinks, $>2$ drinks on any one occasion, $>5$ uses of marijuana, $>4$ cigarettes per day, or any other drug use $[19,20]$. The study was approved by the Oregon Health \& Science University Institutional Review Board.

\section{Baseline participant characteristics}

At baseline, demographic information was collected from adolescents and their parents. To assess socioeconomic status (SES), adolescents' parents were administered the Hollingshead Index of Social Positioning, a measure based on the occupation and educational attainment of each parent [23], with lower scores representing higher SES. To assess intelligence, adolescents were administered the 2-subtest version of the Wechsler Abbreviated Scale of Intelligence [24]. To assess pubertal development, all adolescents completed a modified line drawing version of the Tanner's Sexual Maturation Scale [25], with drawings ranging from 1 (pre-adolescent) to 5 (adult-like maturation). Finally, to evaluate
FH of alcohol use disorder (AUD), parents were administered the Family History Assessment Module [26], which allowed for the calculation of a continuous FH density (FHD) score [17]. FHD was based on the number of adolescents' relatives with an AUD; parents contributed 0.5 each, grandparents 0.25 each, and aunts and uncles a weighted ration of 0.25 divided by their number of siblings, with higher scores indicating greater prevalence of familial AUD. All baseline demographic variables were examined for outliers ( $>2.5 \mathrm{SD}$ from the mean) and normal distribution and were compared between future binge-drinking adolescents and controls.

Follow-up procedure and binge-drinking criterion Following the baseline visit, ongoing quarterly follow-up phone interviews were conducted with adolescents (until age 21), during which adolescents' drug and alcohol use was assessed via a 90-day Timeline Followback [27]. Binge-drinking criterion during these follow-up interviews was three or more reported occasions of binge drinking ( $\geq 5$ drinks for males or $\geq 4$ for females in one episode) within the last 90 days, in acoordance with NIAAA guidelines of binge drinking [28] and consistent with previous studies $[19,20]$. Once an adolescent reached binge-drinking criterion (average age of onset $\sim 17.6$ years), they were matched (based on age, sex, and time since baseline) to a control adolescent (who had not exceeded baseline drug and alcohol use criteria) and both were brought in for reassessment an additional 1-2 times (average 1.9 years between scans; range of 1-6 years). Adolescents were asked to refrain from alcohol use for a minimum of $48 \mathrm{~h}$ prior to their visit (average time since last drink $=18$ days). Additional controls were brought in for re-assessment as part of an ongoing investigation of sex differences in adolescent neurodevelopment [29]. This design resulted in a total of 246 visits among 45 future binge-drinking adolescents and 68 controls. Adolescents were ages 13-20 years (mean $=16.4, n=104$ ) at first reassessment, and ages 15-20 years (mean $=18.0, n=29$ ) at second resassessment.

\section{Image acquisition}

During all visits, participants were scanned on a $3 \mathrm{~T}$ Siemens Magnetom Tim Trio with a 12-channel head coil. DWI scans were collected using a whole-brain, high-angular resolution, echoplanar imaging sequence (repetition time $=9100 \mathrm{~ms}$, echo time $=88 \mathrm{~ms}$, field of view $=256 \mathrm{~mm}^{2}$, slices $=72$, slice thickness $=2 \mathrm{~mm}$ ). Gradient encoding pulses were applied in 30 directions with a $b$-value of $1000 \mathrm{~s} / \mathrm{mm}^{2}$, with six additional images collected with a $b$-value of $0 \mathrm{~s} / \mathrm{mm}^{2}$. Participants received either three $(n=168$; scan time $=16: 52)$ or two $(n=88$; scan time $=11: 24)$ DWI runs. A diffusion field map was also acquired $(T R=790 \mathrm{~ms}$, TE1 $=5.19 \mathrm{~ms}$, $\mathrm{TE} 2=7.65 \mathrm{~ms}$, flip angle $=60^{\circ}, \mathrm{FOV}=240 \mathrm{~mm}^{2}$, slices $=72$, slice thickness $=2 \mathrm{~mm}$, scan time $=3: 13$ ).

\section{Image processing}

Quality assessment and volume censoring. Prior to image processing, all DWI runs underwent strict visual inspection for motion and scanner-related artifacts, as described previously [17, 30], and four image quality metrics (temporal signal-to-noise, mean voxel outlier count, maximum voxel outlier count, and mean relative motion) were obtained. To reduce artifact-induced bias, volumes containing motion or scanner-related artifact were censored; however, to avoid overestimation of diffusion metrics [31], if the same direction/volume was removed from all DWI runs for an individual scan, that scan was excluded from further analyses [17]. As such, 10 scans were excluded for excessive motion, 5 for scanner-artifact, and 2 for errors in image acquisition (i.e. incomplete whole-brain coverage), resulting in a final sample of 109 individuals with 229 total scans. This procedure resulted in significant improvements in all four image quality metrics of scans with one or more censored volumes $(n=124$, all $t \geq 2.481, p<$ 
0.05). Image quality metrics after censoring were not associated with future binge-drinking status or FHD.

Diffusion metrics. DWI data were processed using a combination of FSL (v. 5.0.9) and AFNI (v. 17.1.03), as described previously [17]. Briefly, this consisted of affine registration of the diffusion field map to each DWI run [32], concatenation of all DWI runs within a scan, correction for eddy current distortion, intensity inhomogeneities, head motion [33], and estimation of the diffusion tensor to calculate FA and MD, for each voxel [34].

Image registration. As outlined previously [17, 35], Advanced Normalization Tools (ANTs) algorithms [36] were used to register individual FA maps, first to an unbiased within-subject template and then a study-specific template, before being transformed to Montreal Neurological Institute (MNI) space, in a one-step interpolation. A Gaussian blur (sigma $=1 \mathrm{~mm}$ ) was applied to all FA images after registration [37], and a white matter mask was created that included only voxels where mean FA was greater than 0.3 across all scans. MD maps were registered to standard space using the transformations generated from FA registration.

Group-level analyses

Voxel-wise analyses were carried out for FA/MD using AFNI's 3dLME [38] and fit using Maximum Likelihood (ML), as described previously [17]. A series of baseline and saturated models, including between-individual differences in baseline FA/MD (i.e. random intercepts), were fit and compared voxel-wise using loglikelihood values and the degrees of freedom difference between each model [17]. These model comparisons were thresholded voxel-wise $(p<0.01)$ and corrected for multiple comparisons $(a<$ 0.01) using AFNI's 3dClustsim [39]. To identify white matter regions where $\mathrm{FA} / \mathrm{MD}$ showed significant development with age, a linear growth model (FA/MD Age) was compared to an intercept-only model (FA/MD 1). To assess the effects of future binge-drinking status and FHD on baseline FA/MD only, a maineffects model (FA/MD Age + Binge + FHD) was compared to the linear growth model. Finally, to assess the effects of future bingedrinking status and FHD on both baseline and change in FA/MD, interaction models including future binge-drinking status controlling for FHD (FA/MD Age $\times$ Binge $+F H D)$, FHD controlling for future binge-drinking status (FA/MD Age $\times$ FHD + Binge), and the interaction effect of both FHD and future binge-drinking status $(\mathrm{FA} / \mathrm{MD} \sim$ Age $\times$ Binge $\times \mathrm{FHD})$ were independently compared to the linear growth model. If any region(s) identified in these interaction models overlapped with region(s) identified in the main-effects model (or a simpler interaction model), these models were compared voxel-wise to determine the best fitting, most parsimonious model in a mask containing all significant voxels from both models $(p<0.01 / a<0.01)$. This method of model comparison allows for the interpretation of the fixed effects only after first assessing model fit and providing comparisons to alternative (reduced) models [40, 41].

\section{Post-hoc analyses}

Individual FA and MD values were extracted from all significant regions for post-hoc analyses using the nlme package [42] in $R$ (v. 3.4.2). Prior to modeling, age was centered at age 14 (approximate median baseline age) or age 18 (approximate median follow-up age), and FHD was mean centered. Significant fixed effects were interpreted $(p<0.01)$, and the most parsimonious model was confirmed $(p<0.01)$. For all final models, effect sizes are reported as either Cohen's $d$ (for categorical predictors), standardized regression estimates (for continuous predictors), or the difference between two standardized regression estimates (for interactions between continuous and categorical predictors).

\section{RESULTS}

Participant characteristics

Participant baseline demographics are presented in Table 1, and follow-up alcohol use characteristics in Table S1. While there were no difference in baseline demographics between future bingers and controls, female participants had greater FHD compared to male participants $(U=1940, p<0.05)$, thus sex was tested as a post-hoc covariate in all significant findings.

Age effects (FA/MD Age)

There was a single widespread cluster of FA (283,866 voxels) and MD (324,028 voxels) where significant age effects were present. In these regions, FA increased $(b=0.006, p<0.001, \beta=0.655)$ and MD decreased $(b=-4.13 \mathrm{E}-06, p<0.001, \beta=-0.440)$ with age.

Persistent effects of FHD and/or future binge-drinking (FA/MD Age + Binge + FHD)

There were three regions of $F A$, in the bilateral midbrain/posterior limb of the internal capsule (PLIC) and left cerebellar peduncle, and two regions of $\mathrm{MD}$, in the right $\mathrm{PLIC/thalamus}$ and right superior corona radiata (Table 2), where future binge-drinking adolescents demonstrated persistently greater FA (all $b \geq 0.023, p$ $<0.001, d \geq 0.719$ ) and MD (all $b \geq 1.10 \mathrm{E}-05, p<0.001, d \geq 0.543$ ), compared to controls (Figs. $1 \mathrm{~b}$ and $2 \mathrm{~b}$ ).

Effects of future binge-drinking with age (FA/MD Age $\times$ Binge + FHD)

There were two clusters of FA, in the bilateral PLIC, and one cluster of $\mathrm{MD}$ in the superior corona radiata, where the interaction effect of future binge-drinking with age appeared to overlap with

Table 1. Baseline demographics

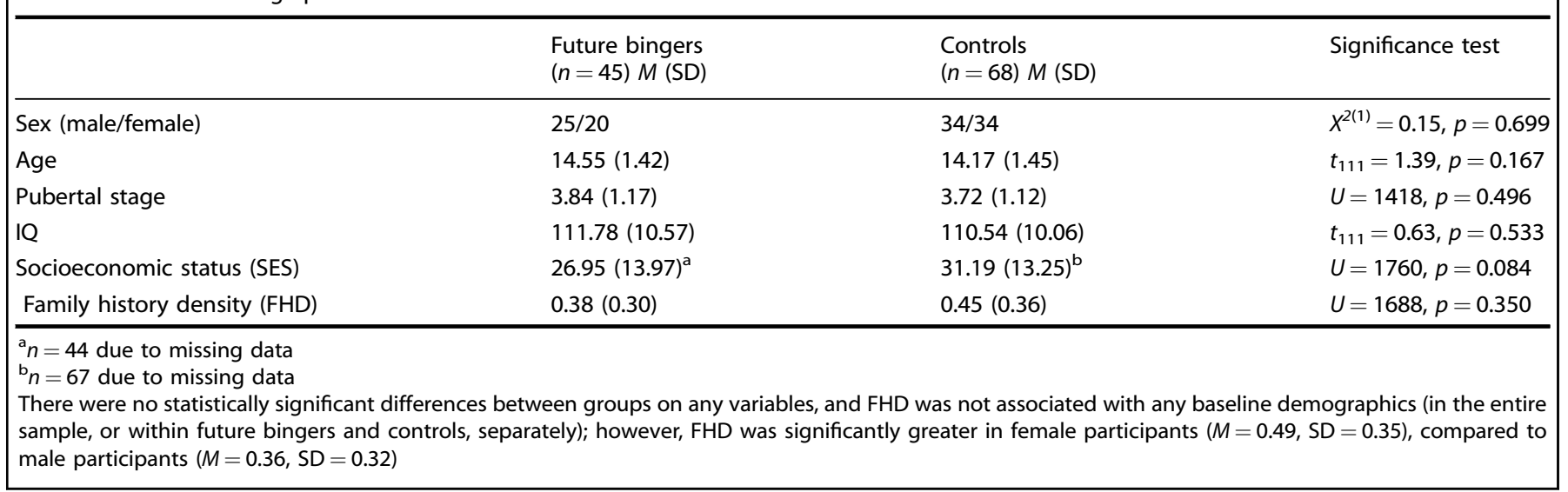


Table 2. Anatomical location of all significant clusters identified in voxel-wise analysis

\begin{tabular}{|c|c|c|c|c|c|}
\hline \multirow[b]{2}{*}{ Anatomical location } & \multicolumn{3}{|c|}{ Peak voxel } & \multirow{2}{*}{$\begin{array}{l}\text { Peak } \\
x^{2}\end{array}$} & \multirow{2}{*}{$\begin{array}{l}\text { Volume } \\
\text { (voxels) }\end{array}$} \\
\hline & $x$ & $y$ & $z$ & & \\
\hline \multicolumn{6}{|l|}{ Fractional anisotropy } \\
\hline \multicolumn{6}{|l|}{$\mathrm{FA} \sim \mathrm{Age}+\mathrm{FHD}+\mathrm{BINGE}$} \\
\hline $\begin{array}{l}\text { Right posterior limb of } \\
\text { the internal capsule }\end{array}$ & -3 & 20 & -20 & 35.111 & 3022 \\
\hline $\begin{array}{l}\text { Left posterior limb of } \\
\text { the internal capsule }\end{array}$ & 7 & 14 & -15 & 27.564 & 2593 \\
\hline $\begin{array}{l}\text { Left cerebellar } \\
\text { peduncle }\end{array}$ & 31 & 45 & -37 & 22.221 & 602 \\
\hline \multicolumn{6}{|l|}{$\mathrm{FA} \sim \mathrm{Age} \times \mathrm{FHD}+\mathrm{BINGE}$} \\
\hline $\begin{array}{l}\text { Left superior frontal } \\
\text { gyrus }\end{array}$ & 20 & -32 & 31 & 23.258 & 540 \\
\hline $\begin{array}{l}\text { Left cerebellar } \\
\text { peduncle }\end{array}$ & 14 & 39 & -30 & $13.587^{\mathrm{a}}$ & 98 \\
\hline \multicolumn{6}{|l|}{$\mathrm{FA} \sim \mathrm{Age} \times \mathrm{FHD} \times \mathrm{BINGE}$} \\
\hline $\begin{array}{l}\text { Splenium of the corpus } \\
\text { callosum }\end{array}$ & 14 & 41 & 15 & 31.606 & 948 \\
\hline \multicolumn{6}{|l|}{ Mean diffusivity } \\
\hline \multicolumn{6}{|l|}{$\mathrm{MD} \sim$ Age $+\mathrm{FHD}+\mathrm{BINGE}$} \\
\hline $\begin{array}{l}\text { Right superior corona } \\
\text { radiata }\end{array}$ & -23 & 23 & 22 & 21.109 & 2022 \\
\hline $\begin{array}{l}\text { Right posterior limb of } \\
\text { the internal capsule }\end{array}$ & -9 & 8 & 8 & 21.521 & 776 \\
\hline \multicolumn{6}{|l|}{$\mathrm{MD} \sim$ Age $\times \mathrm{FHD}+\mathrm{BINGE}$} \\
\hline Right thalamus & -19 & 18 & 10 & $20.150^{b}$ & 274 \\
\hline Right thalamus & -10 & 11 & 13 & $19.879^{b}$ & 69 \\
\hline \multicolumn{6}{|l|}{$\mathrm{MD} \sim$ Age $\times$ BINGE + FHD } \\
\hline $\begin{array}{l}\text { Right superior frontal } \\
\text { gyrus }\end{array}$ & -16 & 11 & 48 & $15.170^{c}$ & 457 \\
\hline Right precentral gyrus & -32 & 12 & 49 & $12.511^{\mathrm{c}}$ & 205 \\
\hline Right precentral gyrus & -36 & 3 & 36 & $15.844^{c}$ & 168 \\
\hline $\begin{array}{l}\text { Right superior } \\
\text { longitudinal fasciculus }\end{array}$ & -35 & 17 & 37 & $10.990^{c}$ & 121 \\
\hline \multicolumn{6}{|l|}{$\mathrm{MD} \sim$ Age $\times \mathrm{FHD} \times \mathrm{BINGE}$} \\
\hline $\begin{array}{l}\text { Right superior frontal } \\
\text { gyrus }\end{array}$ & -17 & -51 & 8 & 40.966 & 1019 \\
\hline
\end{tabular}

FHD family history density, MD mean diffusivity, $F A$ fractional anisotropy Peak $X^{2}$ and volume are reported for each cluster in relation to the linear growth (age-only) model, except as noted:

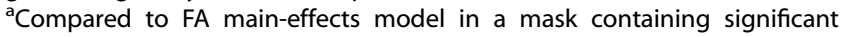
voxels from both models (747 voxels)

${ }^{\mathrm{b}}$ Compared to MD main-effects model in a mask containing significant voxels from both models (1464 voxels)

'Compared to MD main-effects model in a mask containing significant voxels from both models (3708 voxels)

persistent effects of future binge-drinking. Voxel-wise comparisons in these regions found no clusters of FA, and four clusters of $M D$, in the right posterior superior frontal gyrus (SFG), right precentral and right superior longitudinal fasciculus (Table 2), where the interaction effect of future binge-drinking status and age was significant. In these regions, future binge-drinking adolescents had greater MD in early-adolescence (all $b \geq 1.10 \mathrm{E}$ $05, p<0.001, d \geq 0.543$ ), and greater decreases in MD with age (all $b \geq-4.22 \mathrm{E}-06, p<0.01, \Delta \beta \geq-0.346$ ), compared to controls. Thus, no association between future binge drinking and MD was present by late-adolescence (Fig. 2c).
Effects of FHD with age (FA/MD Age $\times$ FHD + Binge)

There were three clusters of FA in the bilateral PLIC and left cerebellar peduncle, and one cluster of MD in the PLIC/thalamus where the interaction effect of FHD with age appeared to overlap with persistent effects of FHD and/or future binge-drinking. Voxelwise comparisons in these regions revealed one cluster of FA in the left cerebellar peduncle and two clusters of $M D$ in the thalamus (Table 2) where the interaction effect of FHD and age was significant. In these regions, future binge-drinking adolescents had persistently greater FA $(b=0.026, p<0.001, d=0.620)$ and MD (all $b \geq 8.03 \mathrm{E}-06, p<0.01, \beta \geq 0.365$ ), compared to controls. Meanwhile, regardless of future binge-drinking, greater FHD was associated with greater FA $(b=0.030, p<0.01, \beta=0.249)$ and lower MD (all $b \geq-1.58 \mathrm{E}-05, p<0.01, \beta \geq-0.225$ ) in earlyadolescence, but greater declines in FA $(b=-0.010, p<0.01$, $\beta=-0.168$ ) and shallower declines in MD (all $b \geq-1.58 \mathrm{E}-05, p<$ $0.01, \beta \geq 0.228$ ) with age. Thus, no association between FHD and FA/MD was present by late-adolescence (Fig. 1d).

Additionally, there was one new cluster of FA identified by this interaction model in the left SFG (Table 2). Here, greater FHD was associated with lower FA in early-adolescence $(b=-0.042$, $p<0.001, \beta=-0.497)$, greater increases in FA with age $(b=0.006$, $p<0.001, \beta=0.148)$, and no association with FA by lateadolescence (Fig. 1c).

Combined effects of future binge drinking and FHD with age (FA/ $\mathrm{MD} \sim$ Age $\times$ Binge $\times \mathrm{FHD}$ )

There were two clusters of FA in the bilateral PLIC, and two clusters of MD in the precentral gyrus and superior corona radiata, identified by this model, which overlapped with previous effects of future binge-drinking status and FHD; however, the interaction model failed to prove significantly better than a reduced model in these regions. Further, this interaction model also revealed one new cluster of FA in the splenium of the corpus callosum and one new cluster of MD in the right SFG (Table 2). In the splenium of the corpus callosum, post-hoc modeling failed to confirm a three-way interaction, and a simplifier model including the interaction effects of future binge-drinking status and FHD, independently (FA Age $\times \mathrm{FHD}+$ Age $\times$ Binge), proved to be the most parsimonious model. In this region, future binge-drinking adolescents, when compared to controls, demonstrated greater FA in earlyadolescence $(b=0.019, p<0.01, d=0.678)$, greater declines in FA with age $(b=-0.003, p<0.01, \Delta \beta=-0.260)$, and comparable levels of FA as controls in late-adolescence. Additionally, greater FHD was associated with reduced FA in early-adolescence $(b=$ $-0.024, p<0.01, \beta=-0.299$ ), but not late-adolescence, regardless of future binge-drinking status. Finally, in the right SFG, there was a significant three-way interaction between future binge-drinking status, FHD and age $(b=2.16 \mathrm{E}-05, p<0.001, \Delta \beta=0.584)$. Early in adolescence, greater FHD was associated with greater MD in control adolescents $(b=2.62 \mathrm{E}-05, p<0.01, \beta=0.346)$, but lower MD in future binge-drinking adolescents $(b=-3.83 \mathrm{E}-05, p<0.01$, $\beta=-0.507)$, while future binge-drinking was associated with overall lower levels of MD $(b=-1.48 \mathrm{E}-05, p<0.01, d=-0.566)$ (Fig. 2e). However, by late-adolescence, there are no effects of FHD or future binge-drinking on MD.

Fixed effects and variance estimates, for all significant clusters, can be found in Tables S2 \& S3. All fixed effects remained significant when controlling post-hoc for sex.

\section{DISCUSSION}

This study is the first to use a longitudinal design to investigate the combined effects of future binge drinking and $\mathrm{FH}$ on white matter microstructural development. As hypothesized, future binge drinking and $\mathrm{FH}$ of alcoholism were associated with altered white matter microstructure in several frontostriatal white matter regions that appear to coincide with tracts that originate in 
A

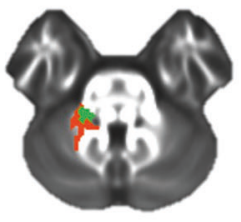

$z=\mathbf{- 3 0}$

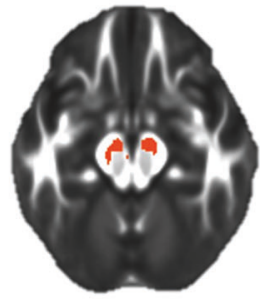

$Z=-15$

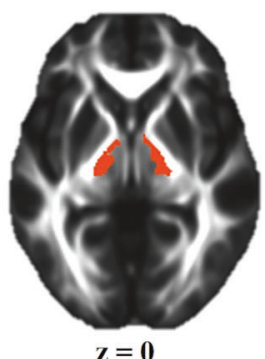

$\mathrm{z}=\mathbf{0}$

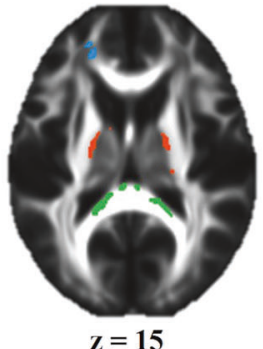

$\mathrm{z}=15$

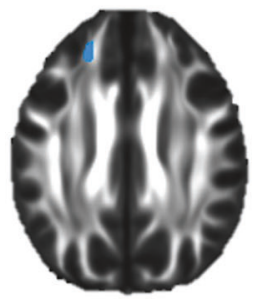

$\mathrm{z}=\mathbf{3 0}$
B

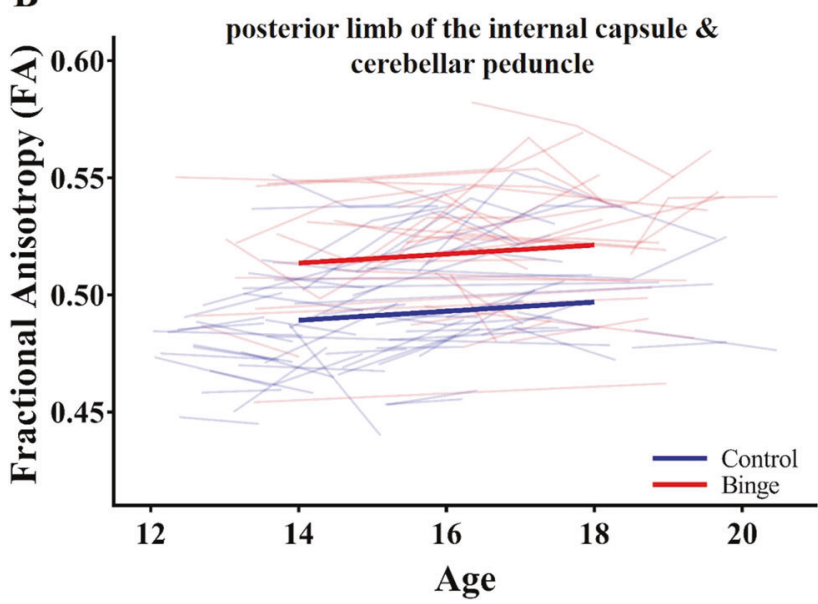

D

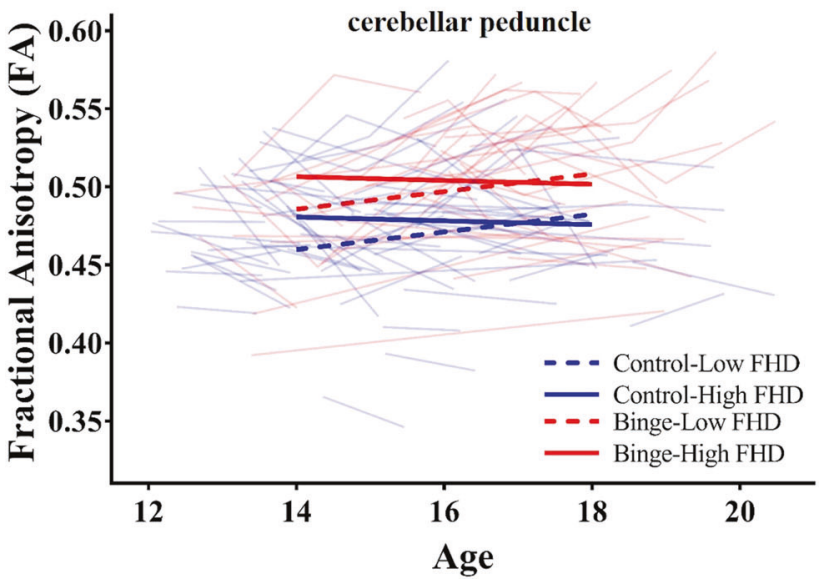

C

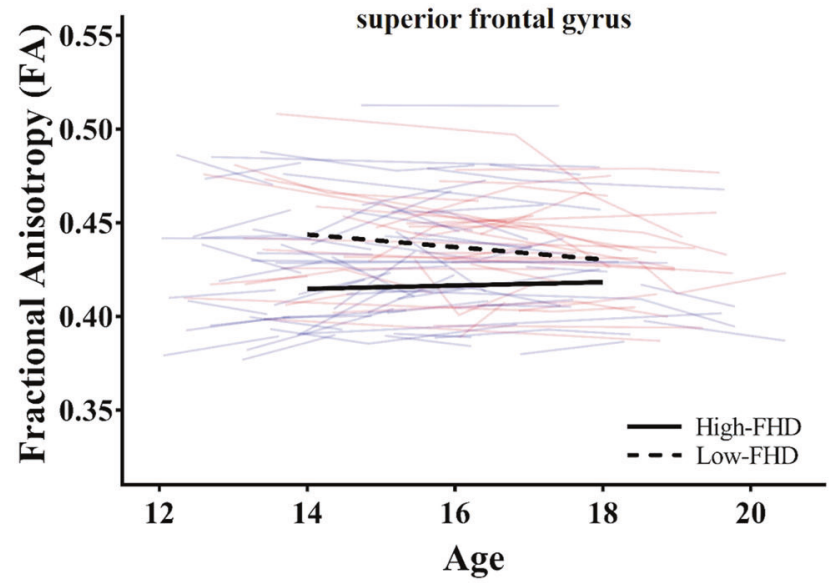

$\mathbf{E}$

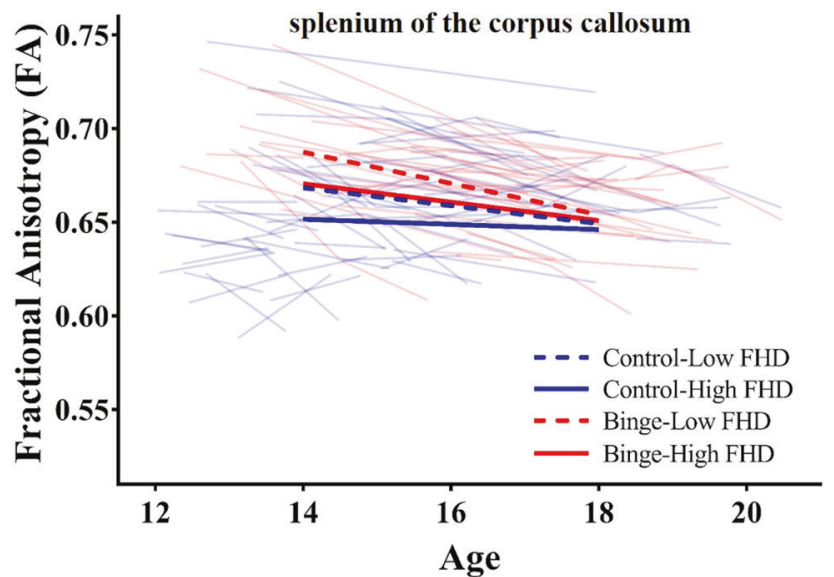

Fig. 1 Effects of future binge-drinking status and family history density (FHD) on fractional anisotropy (FA). a Regions where future bingedrinking status (orange), FHD (blue) or both (green) were associated with altered FA. $\mathbf{b}$ In the posterior limb of the internal capsule and cerebellar peduncle, future binge-drinking status was associated with persistently greater FA with age. $\mathrm{c}$ In the superior frontal gyrus, greater FHD was associated with reduced FA in early-adolescence, but not late-adolescence. $\mathbf{d}$ In a smaller region of the cerebellar peduncle, future binge-drinking status was associated with persistently greater FA, and FHD was associated with reduced FA in early-adolescence but not lateadolescence. $\mathbf{e}$ In the splenium of the corpus callosum, early in adolescence, future binge-drinking status was associated with greater FA, while greater FHD was associated with reduced FA, effects that both dissipated by late-adolescence

midbrain structures, such as the ventral tegmental area, substantia nigra, and thalamus, and extend superiorly past the striatum and nucleus accumbens to the premotor cortex and SFG (Fig. 3). These regions are thought to include several pathways important for the formation of addiction, including the midbrain-striatal-thalamic pathway, important for the binge/intoxication phase, and prefrontal pathways important for alcohol craving [43].

With regard to future binge drinking, contrary to previous longitudinal studies [10, 11], we found that all significant associations with future binge drinking (both persistent and transient) were present prior to alcohol use. This is in line with other structural neuroimaging studies that demonstrate less gray matter volume and thickness, and white matter volume, prior to alcohol use in adolescents that go on to binge-drink [44, 45]. Further, we found that FA was greater in future binge-drinking adolescents than controls. As such, greater FA in white matter regions in the midbrain and internal capsule may represent a predisposition to engage in future alcohol use, while the lower FA previously observed in binge-drinking adolescents $[10,11]$ may be due to the neurotoxic effects of alcohol. This suggests that as adolescents initiate and potentially escalate use, they may demonstrate reductions in FA later in life. 
A

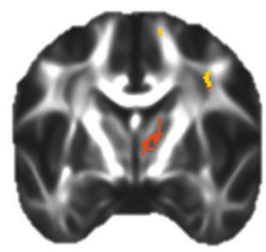

$\mathbf{y}=\mathbf{5}$

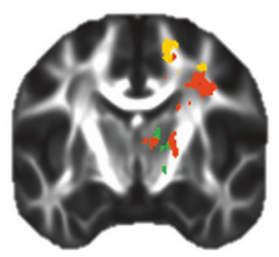

$y=10$

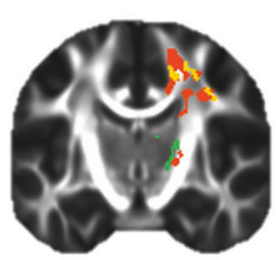

$y=15$

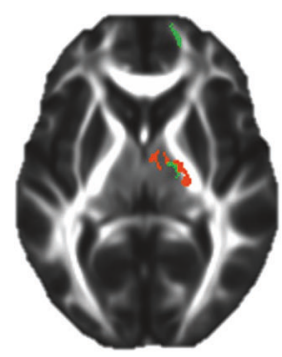

$\mathrm{z}=\mathbf{5}$

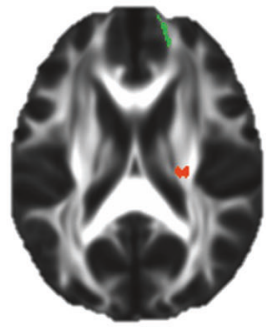

$\mathrm{z}=\mathbf{2 0}$
B

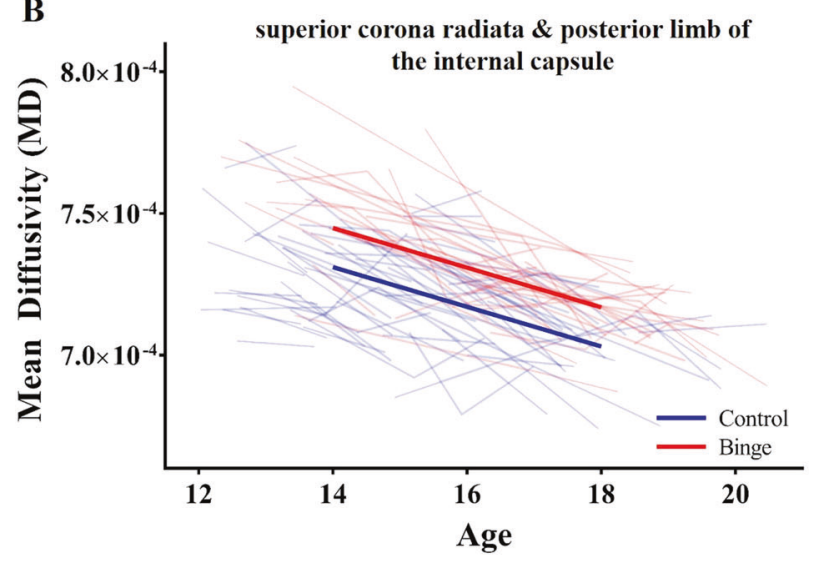

D

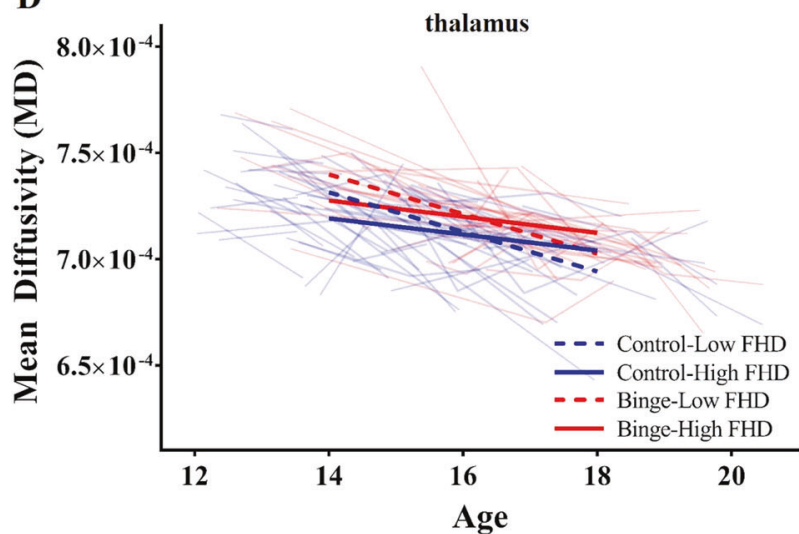

C

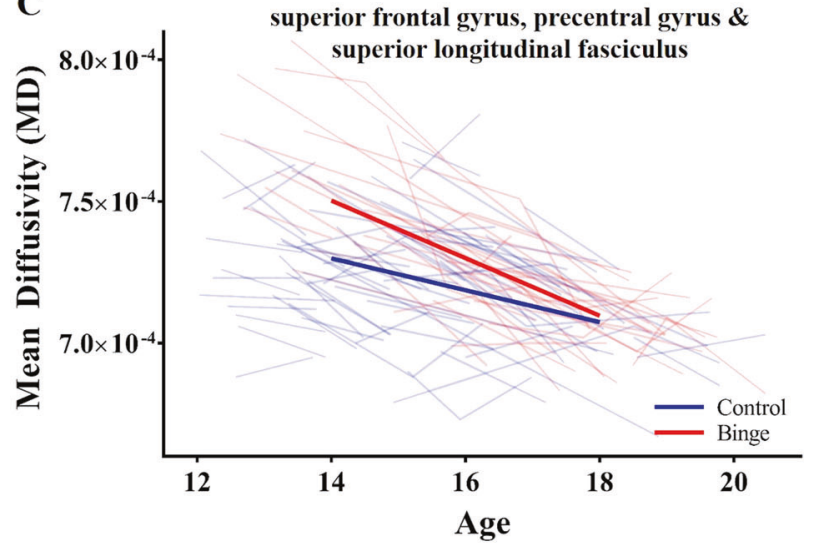

E

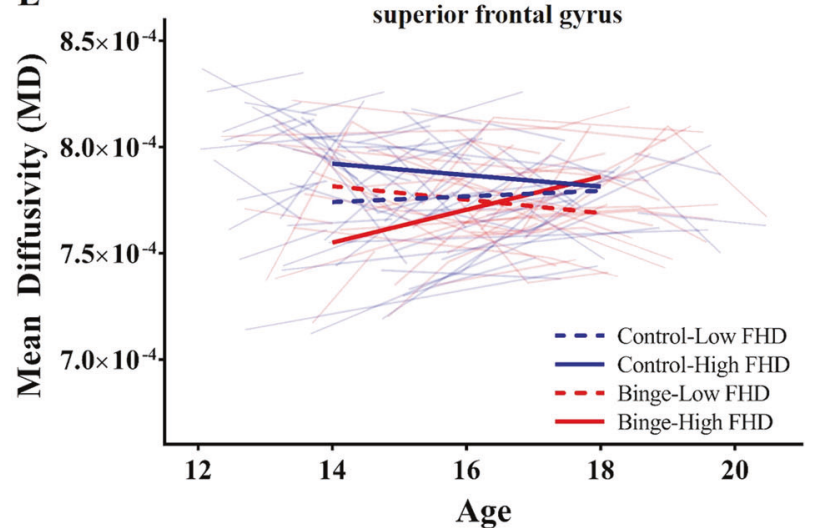

Fig. 2 Effects of future binge-drinking status and family history density (FHD) on mean diffusivity (MD). a Regions of the brain where future binge-drinking status was persistently (orange) and transiently (yellow) associated with altered MD, and regions where both future bingedrinking status and FHD were associated with altered MD (green). $\mathbf{b}$ In the superior corona radiata and posterior limb of the internal capsule, future binge-drinking status was associated with persistently greater MD. c In the superior frontal gyrus, precentral gyrus, and superior longitudinal fasciculus, future binge-drinking status was associated with greater MD in early-adolescence but not late-adolescence. $\mathbf{d}$ In the thalamus, future binge-drinking status was associated with greater MD throughout adolescence, while greater FHD was associated with less MD in early-adolescence but not late-adolescence. e In the superior frontal gyrus, greater FHD was associated with lower MD in future bingedrinking adolescence, and greater MD in control adolescence, early in adolescence; however, this effects dissipated by late-adolescence

Furthermore, this study revealed that both FA and MD were greater in future binge-drinking adolescents than controls. While this is consistent with studies that found binge-drinking adolescents had greater FA in the internal capsule [9] and greater MD in the thalamus, corona radiata, and superior longitudinal fasciculus [11], it runs contrary to typical findings where FA and MD are inversely related. However, despite altered FA and MD occurring in some of the same white matter regions (e.g., PLIC and SFG), they share very little overlap (Fig. 3). Further, in the internal capsule, alterations in FA were evident primarily near subcortical regions, coinciding with white matter tracts thought to interconnect midbrain and reward-relevant regions, such as the cerebellar peduncles, substantia nigra, ventral tegmental area, thalamus, striatum, and nucleus accumbens. Meanwhile, alterations in MD were limited to predominately more cortical regions, coinciding with tracts thought to connect the aforementioned reward-relevant regions to the frontal cortex. This may suggest that early in adolescence, over maturation (greater FA) in striatal white matter regions surrounding reward-relevant regions of the brain, as well as under maturation (greater MD) in frontal white matter regions implicated in cognitive control [46], may predict which adolescents are more likely to go on to binge-drink.

Regarding $\mathrm{FH}$ of alcoholism, this study replicated findings of reduced FA in the prefrontal cortex [14] and reduced MD in the thalamus [13] in association with $\mathrm{FH}$, and further strengthens the notion that the association between $\mathrm{FH}$ and FA/MD is greater in early-adolescence than late-adolescence $[16,17]$. This suggests that adolescents may eventually age-out of some FH-related 


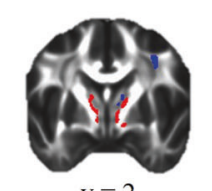

$y=2$

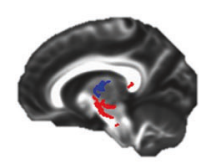

$\mathrm{x}=10$

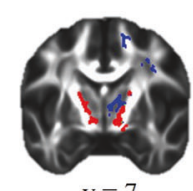

$\mathrm{y}=7$

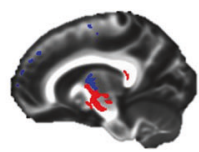

$\mathrm{x}=12$

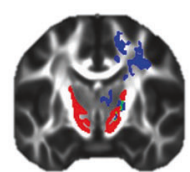

$\mathrm{y}=12$

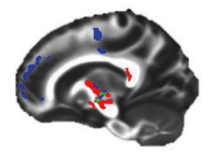

$\mathrm{x}=14$

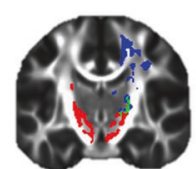

$y=17$

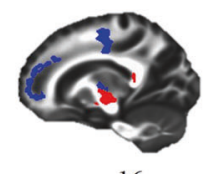

$x=16$

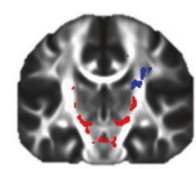

$y=22$

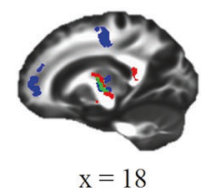

Fig. 3 Frontostriatal regions identified in whole-brain analysis. Out of approximately 13,000 voxels shown to have associations between family history density (FHD) and/or future binge-drinking status and fractional anisotropy (red) or mean diffusivity (blue), only 287 voxels ( 2.2\%; green) had alterations in both

vulnerabilities, even if they engage in binge-drinking behavior. This is further supported by the three-way interaction between future binge-drinking status, FHD and age in the SFG, where greater FHD was associated with greater $\mathrm{MD}$ in control adolescents, and lower MD in future binge-drinking adolescents, prior to alcohol use, but that all effects dissipated by lateadolescence. Altogether, these findings suggest that future bingedrinking adolescents demonstrate a pattern of altered white matter microstructure prior to alcohol use, some of which is related to familial alcoholism. However, this effect is largely transient, with future binge-drinking adolescents and those with familial alcoholism having developmental trajectories that lead to white matter microstructure comparable to those that are largely alcohol-naive in late-adolescence. This finding supports recent hypotheses that adolescence is a period in which the brain is highly adaptive and potentially capable of demonstrating resilience to genetic, environmental, and neurobiological risk phenotypes that may predispose an adolescent more likely to engage in risky behavior such as binge drinking $[47,48]$.

While these findings provide novel insight into the association between personal and familial alcohol misuse and white matter microstructure, limitations should be considered. First, due to sample size and the limited number of within-subject time points, this study was not equipped to investigate other important predictive variables, such as sex [49], or investigate the potential of non-linear white matter microstructural development $[2,5]$. Future studies will be necessary to address these questions. Similarly, given the broad baseline age range, we were unable to appropriately assess the association between age of onset of alcohol use and white matter development. Given previous findings suggesting that functional neurobiology (e.g., ventral striatal brain response during decision making) predicts age of onset of alcohol use [50], future investigations of white matter microstructural predictors of age of onset of alcohol use will be important for confirming our findings regarding neurobiological markers of future alcohol use. Lastly, it cannot be ruled out that control participants in this study (particularly those still in midadolescence during reassessment) will not initiate alcohol use at a later time, or that adolescents currently binge drinking may subsequently increase in or abstain from alcohol use later in life, possibilities that must be taken into account when considering these findings as risk markers for future alcohol misuse.

In conclusion, these findings demonstrate that both $\mathrm{FH}$ of alcoholism and future binge drinking are associated with altered frontostriatal FA (surrounding subcortical regions), and MD (in fronto-cortical regions), in early-adolescence, prior to alcohol use. However, many of these alterations are transient and dissipate by late-adolescence. This suggests that altered maturation of white matter fibers in regions connecting midbrain, striatal and frontal regions important for the development of addiction are present prior to alcohol use in adolescents who later go on to binge-drink, and may be associated at least partially with familial alcoholism. Despite this, adolescence may undergo adaptive neurodevelopment in many of these same regions. These findings are encouraging for the development of future prevention and intervention strategies in adolescence, and may provide useful neurobiological markers for identifying adolescents prone to initiate alcohol use.

\section{ACKNOWLEDGEMENTS}

We would like to acknowledge past and present members of the Developmental Brain Imaging Lab for their assistance in participant recruitment, scheduling, and data collection.

\section{FUNDING AND DISCLOSURE}

This work was supported by the National Institute on Alcohol Abuse and Alcoholism (R01 AA017664 - B.J.N.). The authors declare no competing interests.

\section{ADDITIONAL INFORMATION}

Supplementary Information accompanies this paper at (https://doi.org/10.1038/ s41386-019-0315-x).

Publisher's note: Springer Nature remains neutral with regard to jurisdictional claims in published maps and institutional affiliations.

\section{REFERENCES}

1. Giedd JN, Blumenthal J, Jeffries NO, Castellanos FX, Liu H, Zijdenbos A, et al. Brain development during childhood and adolescence: a longitudinal MRI study. Nat Neurosci. 1999;2:861-3.

2. Tamnes CK, Ostby Y, Fjell AM, Westlye LT, Due-Tonnessen P, Walhovd KB. Brain maturation in adolescence and young adulthood: regional age-related changes in cortical thickness and white matter volume and microstructure. Cereb Cortex. 2010;20:534-48. https://doi.org/10.1093/cercor/bhp118

3. Hagmann $P$, Jonasson L, Maeder P, Thiran J-P, Wedeen VJ, Meuli R. Understanding diffusion MR imaging techniques: from scalar diffusion-weighted imaging to diffusion tensor imaging and beyond. Radiographics. 2006;26:S205-S23.

4. Alexander $A L$, Lee JE, Lazar M, Field AS. Diffusion tensor imaging of the brain. neurotherapeutics: the journal of the American Society for Experimental. Neurotherapeutics. 2007;4:316-29. https://doi.org/10.1016/j.nurt.2007.05.011

5. Lebel C, Beaulieu C. Longitudinal development of human brain wiring continues from childhood into adulthood. J Neurosci: Off J Soc Neurosci. 2011;31:10937-47. https://doi.org/10.1523/jneurosci.5302-10.2011

6. Achterberg M, Peper JS, van Duijvenvoorde AC, Mandl RC, Crone EA. Frontostriatal white matter integrity predicts development of delay of gratification: A longitudinal study. J Neurosci. 2016;36:1954-61.

7. Seghete KLM, Herting MM, Nagel BJ. White matter microstructure correlates of inhibition and task-switching in adolescents. Brain Res. 2013;1527:15-28. https:// doi.org/10.1016/j.brainres.2013.06.003

8. Nagy $\mathrm{Z}$, Westerberg $\mathrm{H}$, Klingberg T. Maturation of white matter is associated with the development of cognitive functions during childhood. J Cogn Neurosci. 2004;16:1227-33. 
9. Bava S, Frank LR, McQueeny T, Schweinsburg BC, Schweinsburg AD, Tapert SF. Altered white matter microstructure in adolescent substance users. Psychiatry Res: Neuroimaging. 2009;173:228-37.

10. Luciana M, Collins PF, Muetzel RL, Lim KO. Effects of alcohol use initiation on brain structure in typically developing adolescents. Am J Drug Alcohol Abus. 2013;39:345-55. https://doi.org/10.3109/00952990.2013.837057

11. Bava S, Jacobus J, Thayer RE, Tapert SF. Longitudinal changes in white matter integrity among adolescent substance users. Alcohol Clin Exp Res. 2013;37: E181-9. https://doi.org/10.1111/j.1530-0277.2012.01920.x

12. Jacobus J, McQueeny T, Bava S, Schweinsburg BC, Frank LR, Yang TT, et al. White matter integrity in adolescents with histories of marijuana use and binge drinking. Neurotoxicol Teratol. 2009;31:349-55. https://doi.org/10.1016/j. ntt.2009.07.006

13. Squeglia LM, Jacobus J, Brumback T, Meloy MJ, Tapert SF. White matter integrity in alcohol-naive youth with a family history of alcohol use disorders. Psychol Med. 2014;44:2775-86. https://doi.org/10.1017/s0033291714000609

14. Herting MM, Schwartz D, Mitchell SH, Nagel BJ. Delay discounting behavior and white matter microstructure abnormalities in youth with a family history of alcoholism. Alcohol Clin Exp Res. 2010;34:1590-602. https://doi.org/10.1111/ j.1530-0277.2010.01244.x

15. Dawson DA, Harford TC, Grant BF. Family history as a predictor of alcohol dependence. Alcohol Clin Exp Res. 1992;16:572-5.

16. Acheson A, Wijtenburg SA, Rowland LM, Winkler AM, Gaston F, Mathias CW, et al. Assessment of whole brain white matter integrity in youths and young adults with a family history of substance-use disorders. Hum Brain Mapp. 2014;35:5401-13.

17. Jones SA, Morales AM, Nagel BJ. Resilience to risk for psychopathology: The role of white matter microstructural development in adolescence. Biol Psychiatry: Cogn Neurosci Neuroimaging. 2019. https://doi.org/10.1016/j.bpsc.2018.08.006. (in press)

18. Hill SY, Terwilliger R, McDermott M. White matter microstructure, alcohol exposure, and familial risk for alcohol dependence. Psychiatry Res. 2013;212:43-53. https://doi.org/10.1016/j.pscychresns.2012.11.003

19. Cservenka A, Jones SA, Nagel BJ. Reduced cerebellar brain activity during reward processing in adolescent binge drinkers. Dev Cogn Neurosci. 2015;16:110-20. https://doi.org/10.1016/j.dcn.2015.06.004

20. Jones SA, Cservenka A, Nagel BJ. Binge drinking impacts dorsal striatal response during decision making in adolescents. Neuroimage. 2016;129:378-88. https:// doi.org/10.1016/j.neuroimage.2016.01.044

21. Lucas $C P$, Zhang $H$, Fisher PW, Shaffer D, Regier DA, Narrow WE, et al. The DISC Predictive Scales (DPS): efficiently screening for diagnoses. J Am Acad Child Adolesc Psychiatry. 2001;40:443-9.

22. Oldfield RC. The assessment and analysis of handedness: the Edinburgh inventory. Neuropsychologia. 1971;9:97-113.

23. Hollingshead AB, Redlich FC. Social class and mental illness: Community study. Hoboken, NJ: John Wiley \& Sons Inc., 1958.

24. Wechsler D. Wechsler abbreviated scale of intelligence. San Antionio, TX: Psychological Corporation; 1999.

25. Taylor SJ, Whincup PH, Hindmarsh PC, Lampe F, Odoki K, Cook DG. Performance of a new pubertal self-assessment questionnaire: a preliminary study. Paediatr Perinat Epidemiol. 2001;15:88-94.

26. Rice JP, Reich T, Bucholz KK, Neuman RJ, Fishman R, Rochberg N, et al. Comparison of direct interview and family history diagnoses of alcohol dependence. Alcohol Clin Exp Res. 1995;19:1018-23.

27. Sobell LC, Brown J, Leo Gl, Sobell MB. The reliability of the Alcohol Timeline Followback when administered by telephone and by computer. Drug Alcohol Depend. 1996;42:49-54.

28. National Institute on Alcohol Abuse and Alcoholism. Drinking levels defined. (2018). https://www.niaaa.nih.gov/alcohol-health/overview-alcohol-consumption/ moderate-binge-drinking. Online document.

29. Alarcon G, Cservenka A, Fair DA, Nagel BJ. Sex differences in the neural substrates of spatial working memory during adolescence are not mediated by endogenous testosterone. Brain Res. 2014;1593:40-54. https://doi.org/10.1016/j.brainres.2014.09.057
30. Roalf DR, Quarmley M, Elliott MA, Satterthwaite TD, Vandekar SN, Ruparel K, et al. The impact of quality assurance assessment on diffusion tensor imaging outcomes in a large-scale population-based cohort. Neuroimage. 2016;125:903-19. https://doi.org/10.1016/j.neuroimage.2015.10.068

31. Chen Y, Tymofiyeva O, Hess CP, Xu D. Effects of rejecting diffusion directions on tensor-derived parameters. Neuroimage. 2015;109:160-70. https://doi.org/ 10.1016/j.neuroimage.2015.01.010

32. Saad ZS, Glen DR, Chen G, Beauchamp MS, Desai R, Cox RW. A new method for improving functional-to-structural MRI alignment using local Pearson correlation Neuroimage. 2009;44:839-48. https://doi.org/10.1016/j.neuroimage.2008.09.037

33. Andersson JLR, Sotiropoulos SN. An integrated approach to correction for offresonance effects and subject movement in diffusion MR imaging. Neuroimage. 2016;125:1063-78. https://doi.org/10.1016/j.neuroimage.2015.10.019

34. Smith SM, Jenkinson M, Woolrich MW, Beckmann CF, Behrens TE, Johansen-Berg $\mathrm{H}$, et al. Advances in functional and structural MR image analysis and implementation as FSL. Neuroimage. 2004;23:S208-19. https://doi.org/10.1016/j. neuroimage.2004.07.051

35. Schwarz CG, Reid RI, Gunter JL, Senjem ML, Przybelski SA, Zuk SM, et al. Improved DTI registration allows voxel-based analysis that outperforms tract-based spatial statistics. Neuroimage. 2014;94:65-78.

36. Avants BB, Tustison NJ, Song G, Cook PA, Klein A, Gee JC. A Reproducible evaluation of ANTs similarity metric performance in brain image registration. Neuroimage. 2011;54:2033-44.

37. Ashburner J, Friston KJ. Voxel-based morphometry-the methods. Neuroimage. 2000;11:805-21. https://doi.org/10.1006/nimg.2000.0582

38. Chen G, Saad ZS, Britton JC, Pine DS, Cox RW. Linear mixed-effects modeling approach to FMRI group analysis. Neuroimage. 2013;73:176-90. https://doi.org/ 10.1016/j.neuroimage.2013.01.047

39. Forman SD, Cohen JD, Fitzgerald M, Eddy WF, Mintun MA, Noll DC. Improved assessment of significant activation in functional magnetic resonance imaging (fMRI): use of a cluster-size threshold. Magn Reson Med: Off J Soc Magn Reson Med / Soc Magn Reson Med. 1995;33:636-47.

40. Singer JD, Willett JB. Applied longitudinal data analysis: Modeling change and event occurrence. New York, NY: Oxford University Press; 2003.

41. Bliese PD, Ployhart RE. Growth modeling using random coefficient models: Model building, testing, and illustrations. Organ Res Methods. 2002;5:362-87.

42. Pinheiro J, Bates D, DebRoy S, Sarkar D, Team RC. Linear and nonlinear mixed effects models. R Package Version. 2009;3:1-117.

43. Koob GF, Volkow ND. Neurocircuitry of addiction. Neuropsychopharmacol: Off Publ Am Coll Neuropsychopharmacol. 2010;35:217.

44. Squeglia LM, Rinker DA, Bartsch $H$, Castro N, Chung $Y$, Dale AM, et al. Brain volume reductions in adolescent heavy drinkers. Dev Cogn Neurosci. 2014;9:117-25. https://doi.org/10.1016/j.dcn.2014.02.005

45. Squeglia LM, Tapert SF, Sullivan EV, Jacobus J, Meloy MJ, Rohlfing T, et al. Brain development in heavy-drinking adolescents. Am J Psychiatry. 2015;172:531-42. https://doi.org/10.1176/appi.ajp.2015.14101249

46. Morein-Zamir S, Robbins TW. Fronto-striatal circuits in response-inhibition: Relevance to addiction. Brain Res. 2015;1628:117-29. https://doi.org/10.1016/j. brainres.2014.09.012

47. Sercombe H. Risk, adaptation and the functional teenage brain. Brain Cogn. 2014;89:61-9. https://doi.org/10.1016/j.bandc.2014.01.001

48. Cousijn J, Luijten M, Ewing SWF. Adolescent resilience to addiction: a social plasticity hypothesis. Lancet Child \& Adolesc Health. 2018;2:69-78.

49. Simmonds DJ, Hallquist MN, Asato M, Luna B. Developmental stages and sex differences of white matter and behavioral development through adolescence: A longitudinal diffusion tensor imaging (DTI) study. Neuroimage. 2014;92:356-68. https://doi.org/10.1016/j.neuroimage.2013.12.044

50. Morales AM, Jones SA, Ehlers A, Lavine JB, Nagel BJ. Ventral striatal response during decision making involving risk and reward is associated with future binge drinking in adolescents. Neuropsychopharmacology. 2018. https://doi.org/ $10.1038 /$ s41386-018-0087-8 